Acta Math. Acad. Sci. Hung. 8 (1957), pp. 107-126.

\title{
ON ADDITIVE AND MULTIPLICATIVE TOTALS
}

\author{
András Prékopa (Budapest) \\ (Presented by A. Rényi) \\ Mathematical Institute of the Hungarian Academy of Sciences
}

Received: October 18, 1956

\section{Introduction}

In the present paper terminology "total" is used for a generalization of the Burkill integral and multiplicative integral, respectively. The functions, the totals of which are considered, take their values from a Banach algebra $\mathcal{B}$ with a unity. This means a Banach space $\mathcal{B}$ in which for every pair $f \in \mathcal{B}, g \in \mathcal{B}$ a product $f g \in \mathcal{B}$ is defined such that $\|f g\| \leq\|f\|\|g\|$ and if $h \in \mathcal{B}$, then $f(g+h)=f g+f h,(g+h) f=g f+h f$, finally there is an $e \in \mathcal{B}$ with the properties $e f=f e=f,\|e\|=1$. It is proved that under some conditions the additive (and multiplicative) total of a multiplicative (and additive, resp.) set function exists. The theorems of this type are useful in solving some functional equations (see e.g. [3] and $\S 6)$ and studying the properties of multiplicative set functions by tracing the problems to those formulated in terms of additive set functions.

The multiplicative integral (on the real axis for matrix-valued functions) has been introduced by V. Volterra [12], [13], [14] and considered by several authors: L. Schlesinger [8], [9], [10], [11], G. Rasch [7], R.L Dobrušin [3]; G. Birkhoff [2] has given a generalization of this integral by using more general notions instead of matrices. The additive integral (on the real axis for matrix-valued functions) has been considered by M. FRÉCHET [4] and R. L. Dobrušin [3]. The theorems proved in the present paper are analogous to those of DoBRušin [3] and will be used in the theory of stochastic set functions. ${ }^{1}$ In $\S 6$ we anticipate an example from this as in this special case (Example 1) the relevant statements can be proved at once by the aid of the present paper.

\section{$\S 1$. Notions and notations}

Throughout the paper the basic space will be denoted by $H$ which is supposed to be metric and compact. We suppose that we are given a class of sets $\mathcal{K}$ consisting of some subsets of $H$ and satisfying the following conditions:

\footnotetext{
${ }^{1}$ A. PrÉKOPA, On stochastic set functions. III, Acta Math. Acad. Sci. Hung., 8 (1957), 375-400.
} 
a) $\mathcal{K}$ is a semi-ring, i.e. if $A_{1} \in \mathcal{K}, A_{2} \in \mathcal{K}$, then $A_{1} A_{2} \in \mathcal{K}$ and if $A_{1} \subseteq A_{2}$, then there exists a finite number of sets $C_{1}, C_{2}, \ldots, C_{n}$ such that $C_{i} \in \mathcal{K}(i=1,2, \ldots, n)$, $C_{i} C_{k}=0$ if $i \neq k$ and $A_{2}-A_{1}=\sum_{i=1}^{n} C_{i}{ }^{2}$ We suppose furthermore that this can be done always so that $n \leq R$, where $R$ is a positive integer independent of the sets $A_{1}, A_{2}$.

b) If $h \in H$, then $\{h\} \in \mathcal{K}$.

c) If $A \in \mathcal{K}$, then for every positive integer $r$ and every $\varepsilon>0$ there is a decomposition $A_{1}, A_{2}, \ldots, A_{r}$ of the set $A$ into pairwise disjoint sets of $\mathcal{K}$ such that $\max _{1 \leq k \leq r} d\left(A_{k}\right) \leq \varepsilon .^{3}$

A finite sequence of sets $A_{1}, A_{2}, \ldots, A_{r}$, for which $A_{i} \in \mathcal{K}(i=1,2, \ldots, r), A_{i} A_{k}=0$ if $i \neq k$ and $A=\sum_{k=1}^{r} A_{k} \in \mathcal{K}$, will be called a decomposition of the set $A$ (or briefly decomposition) and will be denoted by $\mathfrak{z}=\left\{A_{1}, A_{2}, \ldots, A_{r}\right\}$. If $\mathfrak{z}_{1}=\left\{A_{i}^{(1)}\right\}, \mathfrak{z}_{2}=\left\{A_{i}^{(2)}\right\}$ are two decompositions and every $A_{i}^{(2)}$ can decomposed by means of some $A_{i}^{(1)}$, then we write $\mathfrak{z}_{2} \sqsubset \mathfrak{z}_{1}$. We shall use the following definitions:

Definition 1 Let us suppose that to every decomposition $\mathfrak{z}=\left\{A_{1}, A_{2}, \ldots, A_{r}\right\}$ there corresponds a permutation $\mathcal{P}(\mathfrak{z})=\left(A_{i_{1}}, A_{i_{2}}, \ldots, A_{i_{r}}\right)$ of the sets of $\mathfrak{z}$ such that if $\mathfrak{z}_{1} \sqsubset \mathfrak{z}_{2}$ and $\mathcal{P}\left(\mathfrak{z}_{1}\right)$ is given by $\left(A_{i_{1}}^{(1)}, A_{i_{2}}^{(1)}, \ldots, A_{i_{r}}^{(1)}\right)$, then in $\mathcal{P}\left(\mathfrak{z}_{2}\right)$ first come those sets of $\mathfrak{z}_{2}$ which decompose $A_{i_{1}}^{(1)}$, then those which decompose $A_{i_{2}}^{(1)}$ etc.

A correspondence between the decompositions and permutations described above will be called a permutation function.

Definition 2 Let $f(A)(A \in \mathcal{K})$ be a set function with values in the Banach algebra $\mathcal{B}$. If for every pair $A_{1}, A_{2}$ of disjoint sets of $\mathcal{K}$, for which $A=A_{1}+A_{2} \in \mathcal{K}$, the relation

$$
f(A)=f\left(A_{1}\right)+f\left(A_{2}\right)
$$

holds, then the set function $f(A)$ will be called additive. $(*)$ implies that $f(0)=0$.

Definition 3 Let $g(A)(A \in \mathcal{K})$ be a set function with values in the Banach algebra $\mathcal{B}$. If there is a permutation function $\mathcal{P}$ such that for every system $A_{1}, A_{2}, \ldots, A_{r}$ of disjoint sets of $\mathcal{K}$, for which $A=\sum_{k=1}^{r} A_{k} \in \mathcal{K}$, the relation

$$
g(A)=\prod_{k=1}^{r} g\left(A_{i_{k}}\right)
$$

holds where $\mathfrak{z}=\left\{A_{1}, A_{2}, \ldots, A_{r}\right\}$ and $\mathcal{P}(\mathfrak{z})=\left(A_{i_{1}}, A_{i_{2}}, \ldots, A_{i_{r}}\right)$, then the set function $g(A)$ will be called multiplicative. We suppose in this case that $f(0)=e$.

\footnotetext{
${ }^{2}$ This notion of semi-rings, which is more general than that of P. HALmos (cf. Measure theory, Chapter 1, $\S 4)$, is due to Á. Császár.

${ }^{3}$ If $B \subseteq H$, then $d(B)$ denotes the diameter of the set $B$, i.e. $d(B)=\sup \rho\left(h_{1}, h_{2}\right)$, where $h_{1} \in B$, $h_{2} \in B$ and $\rho\left(h_{1}, h_{2}\right)$ is the distance between $h_{1}$ and $h_{2}$.
} 
If $\mathcal{B}$ is commutative, then we do not require the existence of a permutation function $\mathcal{P}$. All the statements in this paper are to be taken in the sense that if $\mathcal{B}$ is commutative, then we omit the requirements regarding to the permutation function.

Definition $4 \mathrm{~A}$ set function $\alpha(A)(A \in \mathcal{K})$ with values in $\mathcal{B}$ is called of bounded variation if there is a number $K$ such that for every system $A_{1}, A_{2}, \ldots, A_{r}$ of disjoint sets of $\mathcal{K}$ we have

$$
\sum_{k=1}^{r}\left\|\alpha\left(A_{k}\right)\right\| \leq K
$$

If $A_{i} \subseteq A(i=1,2, \ldots, r)$, then the smallest $K$ for which relation (**) holds will be denoted by $\operatorname{Var}_{\alpha}(A)$.

Definition 5 A set function $\alpha(A)(A \in \mathcal{K})$ with values in $\mathcal{B}$ is said to be $v$-continuous if for every sequence $B_{1}, B_{2}, \ldots$ of sets of $\mathcal{K}$, for which $\lim _{k \rightarrow \infty} B_{k}=0$ and $\lim _{k \rightarrow \infty} d\left(B_{k}\right)=0$, the relation

$$
\lim _{k \rightarrow \infty} \operatorname{Var}_{\alpha}\left(B_{k}\right)=0
$$

holds.

Definition 6 Let $\alpha(A)(A \in \mathcal{K})$ be a set function with values in $\mathcal{B}$. Suppose that there exists a $\beta(B)$ such that for every $\varepsilon>0$ a number $\delta>0$ can be found with the property

$$
\left\|\sum_{k=1}^{r} \alpha\left(A_{k}\right)-\beta(B)\right\| \leq \varepsilon,
$$

provided that $\max _{1 \leq k \leq r} d\left(A_{k}\right) \leq \delta$ where $A_{1}, A_{2}, \ldots, A_{r}$ is a decomposition of the set $B \in \mathcal{K}$. In this case we say that the additive total of $\alpha(A)$ exists in $B$ and we denote it by

$$
\beta(B)=\mathrm{S}_{B} \alpha(d A)
$$

Definition 7 Let $\alpha(A)(A \in \mathcal{K})$ be a set function with values in $\mathcal{B}$. Suppose that there exists a permutation function $\mathcal{P}$ and a $\gamma(B) \in \mathcal{B}$ such that for every $\varepsilon>0$ a number $\delta>0$ can be found with the property

$$
\left\|\prod_{k=1}^{r} \alpha\left(A_{i_{k}}\right)-\gamma(B)\right\| \leq \varepsilon,
$$

provided that $\max _{1 \leq k \leq r} d\left(A_{k}\right) \leq \delta$ where $\left(A_{i_{1}}, A_{i_{2}}, \ldots, A_{i_{r}}\right)$ is the permutation corresponding to the decomposition $\mathfrak{z}=\left\{A_{1}, A_{2}, \ldots, A_{r}\right\}$ of the set $B \in \mathcal{K}$. In this case we say that the multiplicative total of $\alpha(A)$ exists in $B$ relative to the permutation function $\mathcal{P}$. This total will be denoted by

$$
\gamma(B)={ }_{\mathcal{P}} \prod_{B} \alpha(d A)
$$


It is easy to see that both totals are uniquely determined and

$$
\begin{gathered}
\mathrm{S}_{A_{1}+A_{2}} \alpha(d A)=\mathrm{S}_{A_{1}} \alpha(d A)+\mathrm{S}_{A_{2} \alpha(d A),} \\
{ }_{\mathcal{P}} \prod_{A_{1}+A_{2}+\ldots+A_{r} \alpha} \alpha(d A)={ }_{\mathcal{P}} \prod_{A_{i_{1}} \alpha(d A) \cdot{ }_{\mathcal{P}}} \prod_{A_{i_{2}} \alpha(d A) \cdots{ }_{\mathcal{P}}} \prod_{A_{i_{r}} \alpha(d A),}
\end{gathered}
$$

where $A_{i} \in \mathcal{K}(i=1,2, \ldots, r), A_{i} A_{k}=0$ if $i \neq k$ and $\left(A_{i_{1}}, A_{i_{2}}, \ldots, A_{i_{r}}\right)$ is the permutation given by $\mathcal{P}$, provided that the totals on both sides exist. If $\alpha_{1}(A)$ and $\alpha_{2}(A)$ are two set

functions defined on $\mathcal{K}$ and both are additively totalizable in $B$, then the same holds for $\alpha(A)=c_{1} \alpha_{1}(A)+c_{2} \alpha_{2}(A)$ and

$$
\mathrm{S}_{B} \alpha(d A)=c_{1} \mathrm{~S}_{B} \alpha_{1}(d A)+c_{2} \mathbf{S}_{B} \alpha_{2}(d A),
$$

where $c_{1}, c_{2}$ are constants. An analogous relation holds also for the multiplicative total if $\mathcal{B}$ is commutative. In this case if the multiplicative totals of $\alpha_{1}(A)$ and $\alpha_{2}(A)$ exist in $B \in \mathcal{K}$, then that of $\alpha(A)=\alpha_{1}(A) \alpha_{2}(A)$ in $B$ exists too and

$$
\prod_{B} \alpha(d A)=\prod_{B} \alpha_{1}(d A) \prod_{B} \alpha_{2}(d A) .
$$

\section{$\S$ 2. Preliminary lemmas}

In this $\S$ we prove some simple inequalities for Banach algebras and lemmas for additive and multiplicative set functions.

Lemma 1 If $f_{i} \in \mathcal{B}, g_{i} \in \mathcal{B}(i=1,2, \ldots, n)$ and

$$
\left\|\prod_{i=1}^{j} f_{i}\right\| \leq K, \quad\left\|\prod_{i=j}^{n} g_{i}\right\| \leq K \quad(j=1,2, \ldots, n)
$$

where $K$ is a constant, then

$$
\left\|\prod_{i=1}^{n} f_{i}-\prod_{i=1}^{n} g_{i}\right\| \leq K^{2} \sum_{i=1}^{n}\left\|f_{i}-g_{i}\right\| .
$$

Proof. Since

$$
\prod_{i=1}^{n} f_{i}-\prod_{i=1}^{n} g_{i}=\sum_{i=1}^{n} f_{1} \ldots f_{i-1}\left(f_{i}-g_{i}\right) g_{i+1} \ldots g_{n}
$$

it follows that

$$
\left\|\prod_{i=1}^{n} f_{i}-\prod_{i=1}^{n} g_{i}\right\| \leq \sum_{i=1}^{n}\left\|f_{1} \ldots f_{i-1}\right\|\left\|f_{i}-g_{i}\right\|\left\|g_{i+1} \ldots g_{n}\right\| \leq K^{2} \sum_{i=1}^{n}\left\|f_{i}-g_{i}\right\| .
$$


Lemma 2 Let $f_{1}, f_{2}, \ldots, f_{n}$ be such elements of $\mathcal{B}$ that

$$
\sum_{i=1}^{n}\left\|f_{i}\right\| \leq c<1
$$

In this case if $r<n$, then

$$
\begin{aligned}
& \| \prod_{i=1}^{n}\left(e+f_{i}\right)-\left(e+\sum_{i=1}^{n} f_{i}+\sum_{1 \leq i_{1}<i_{2} \leq n} f_{i_{1}} f_{i_{2}} \ldots f_{i_{r}}+\cdots\right. \\
& \left.\quad+\sum_{1 \leq i_{1}<i_{2} \ldots<i_{r} \leq n} f_{i_{1}} f_{i_{2}} \ldots f_{i_{r}}\right) \| \leq \frac{1}{1-c}\left(\sum_{i=1}^{n}\left\|f_{i}\right\|\right)^{r+1} .
\end{aligned}
$$

Proof. Let us start from the identity

$$
\prod_{i=1}^{n}\left(e+f_{i}\right)=e+\sum_{i=1}^{n} f_{i}+\sum_{1 \leq i_{1}<i_{2} \leq n} f_{i_{1}} f_{i_{2}}+\cdots+f_{1} f_{2} \ldots f_{n} .
$$

It follows from this that

$$
\begin{aligned}
& \| \prod_{i=1}^{n}\left(e+f_{i}\right)-\left(e+\sum_{i=1}^{n} f_{i}+\sum_{1 \leq i_{1}<i_{2} \leq n} f_{i_{1}} f_{i_{2}}+\cdots\right. \\
& \left.\quad+\sum_{1 \leq i_{1}<i_{2} \ldots<i_{r} \leq n} f_{i_{1}} f_{i_{2}} \cdots f_{i_{r}}\right) \| \\
& \quad \leq \sum_{1 \leq i_{1}<i_{2}<\ldots<i_{r+1} \leq n}\left\|f_{i_{1}}\right\|\left\|f_{i_{2}}\right\| \cdots\left\|f_{i_{r+1}}\right\|+\cdots+\left\|f_{1}\right\|\left\|f_{2}\right\| \cdots\left\|f_{n}\right\| \\
& \leq\left(\sum_{i=1}^{n}\left\|f_{i}\right\|\right)^{r+1}+\cdots+\left(\sum_{i=1}^{n}\left\|f_{i}\right\|\right)^{n} \\
& \leq \frac{1}{1-\sum_{i=1}^{n}\left\|f_{i}\right\|}\left(\sum_{i=1}^{n}\left\|f_{i}\right\|\right)^{r+1} \\
& \leq \frac{1}{1-c}\left(\sum_{i=1}^{n}\left\|f_{i}\right\|\right)^{r+1}
\end{aligned}
$$

what was to be proved.

For $r=0$ and $r=1$ we obtain as special cases of Lemma 2 the inequalities

$$
\begin{aligned}
\left\|\prod_{i=1}^{n}\left(e+f_{i}\right)-e\right\| & \leq \frac{1}{1-c} \sum_{i=1}^{n}\left\|f_{i}\right\|, \\
\left\|\prod_{i=1}^{n}\left(e+f_{i}\right)-\left(e+\sum_{i=1}^{n} f_{i}\right)\right\| & \leq \frac{1}{1-c}\left(\sum_{i=1}^{n}\left\|f_{i}\right\|\right)^{2} .
\end{aligned}
$$


Lemma 3 If $\alpha(A)(A \in \mathcal{K})$ is a set function of bounded variation with values in the Banach algebra $\mathcal{B}$, then there exists a countable set $H_{1} \subseteq H$ such that $\alpha(h)=0$ if $h \in$ $H-H_{1}$ (we use the notation $\alpha(h)$ for $\alpha(\{h\})$ ).

Proof. Since $\alpha(A)$ is of bounded variation, the set of those $h$ 's for which $\|\alpha(h)\| \geq \frac{1}{n}$, is finite. If $n$ runs over the positive integers, then we obtain all the points $h$ for which $\|\alpha(h)\|>0$. Thus Lemma 3 is proved.

Lemma 4 Let $\alpha(A),(A \in \mathcal{K})$ be a multiplicative set function with values in the Banach algebra $\mathcal{B}$ for which $K=\operatorname{Var}_{\alpha-e}(H)<\infty$. If $B_{1} \in \mathcal{K}, B_{2} \in \mathcal{K}, B_{1} \subseteq B_{2}$ and $B_{2}-B_{1}=$ $\sum_{k=1}^{r} C_{k}$ where $C_{k} \in \mathcal{K}(k=1,2, \ldots, r)$, then

$$
\operatorname{Var}_{\alpha-e}\left(B_{2}-B_{1}\right) \leq(K+1)^{2 r} \sum_{k=1}^{r} \operatorname{Var}_{\alpha-e}\left(C_{i}\right) \text {. }
$$

Proof. Let $A_{1}, A_{2}, \ldots, A_{n}$ be a system of disjoint sets of $\mathcal{K}$,

$$
\sum_{k=1}^{n} A_{k} \subseteq B_{2}-B_{1}
$$

In this case

$$
A_{k}=\sum_{i=1}^{r} A_{k} C_{i} \quad(k=1,2, \ldots, n) .
$$

If $\mathfrak{z}_{k}=\left\{A_{k} C_{1}, A_{k} C_{2}, \ldots, A_{k} C_{r}\right\}$ and $\mathcal{P}\left(\mathfrak{z}_{k}\right)=\left(A_{k} C_{i_{1}}, A_{k} C_{i_{2}}, \ldots, A_{k} C_{i_{r}}\right)$, then

$$
\alpha\left(A_{k}\right)=\prod_{l=1}^{r} \alpha\left(A_{k} C_{i_{l}}\right) .
$$

Since $\|\alpha(B)\| \leq\|\alpha(B)-e\|+1 \leq K+1(B \in \mathcal{K})$, it follows that

$$
\left.\begin{array}{l}
\left\|\prod_{l=r_{1}}^{r} \alpha\left(A_{k} C_{i_{l}}\right)\right\| \leq(K+1)^{r}, \\
\left\|\prod_{l=1}^{r_{1}} \alpha\left(A_{k} C_{i_{l}}\right)\right\| \leq(K+1)^{r},
\end{array}\right\} \quad\left(1 \leq r_{1} \leq r\right) .
$$

Hence, applying the inequality (1), we get

$$
\sum_{k=1}^{n}\left\|e-\alpha\left(A_{k}\right)\right\| \leq \sum_{k=1}^{n}(K+1)^{2 r} \sum_{l=1}^{r}\left\|e-\alpha\left(A_{k} C_{l}\right)\right\| \leq(K+1)^{2 r} \sum_{l=1}^{r} \operatorname{Var}_{\alpha-e}\left(C_{l}\right) .
$$

Thus Lemma 4 is proved.

If $\alpha$ is additive, then we have the stronger relation

$$
\operatorname{Var}_{\alpha}\left(B_{2}-B_{1}\right) \leq \sum_{k=1}^{r} \operatorname{Var}_{\alpha}\left(C_{i}\right)
$$


Lemma 5 Let us suppose that the set function $\alpha(A)(A \in \mathcal{K})$ with values in the Banach algebra $\mathcal{B}$ is multiplicative (and additive, resp.), $K=\operatorname{Var}_{\alpha-\alpha(0)}(H)<\infty$ and $\alpha(A)-\alpha(0)$ is $v$-continuous. Then for every $\varepsilon>0$ there can be found $a \delta>0$ such that if $U \in \mathcal{K}$ is a set with $d(U) \leq \delta$ and $\|\alpha(h)-\alpha(0)\| \leq \varepsilon$ for $h \in U$, then

$$
\operatorname{Var}_{\alpha-\alpha(0)}(U) \leq 2(K+1)^{2 R} \varepsilon \text {. }
$$

Proof. Contrary to the assertion let us suppose that there exists an $\varepsilon_{0}>0$ and a sequence of sets $U_{k}$ for which

$$
\lim _{k \rightarrow \infty} d\left(U_{k}\right)=0, \quad\|\alpha(h)-\alpha(0)\| \leq \varepsilon_{0}
$$

if $h \in \sum_{k=1}^{\infty} U_{k}$ and

$$
\operatorname{Var}_{\alpha-\alpha(0)}\left(U_{k}\right)>2(K+1)^{2 R} \varepsilon_{0}
$$

Since $H$ is a compact metric space, we may suppose without restricting the generality that all the sequences $h_{k}$, where $h_{k} \in U_{k}(k=1,2, \ldots)$, are convergent. Let $h^{\prime}$ denote their common limit element. We can distinguish two cases.

In the first case $h^{\prime}$ is contained at most in a finite number of sets $U_{n}$. This implies that $\lim _{n \rightarrow \infty} U_{n}=0$ and thus, since $\alpha-\alpha(0)$ is $v$-continuous,

$$
\lim _{n \rightarrow \infty} \operatorname{Var}_{\alpha-\alpha(0)}\left(U_{n}\right)=0
$$

which contradicts (6).

In the second case, if $h^{\prime}$ is contained in infinitely many $U_{n}$, then we choose a number $N$ such that

$$
\operatorname{Var}_{\alpha-\alpha(0)}\left(U_{N}-h^{\prime}\right)<\varepsilon_{0} .
$$

The possibility of this assured by Lemma 4 and the $v$-continuity of $\alpha-\alpha(0)$. According to (6) there exists in $U_{N}$ a system of disjoint sets $A_{1}, A_{2}, \ldots, A_{r}$ that satisfies the inequality

$$
\sum_{k=1}^{r}\left\|\alpha\left(A_{k}\right)-\alpha(0)\right\|>2(K+1)^{2 R} \varepsilon_{0}
$$

The element $h^{\prime}$ is contained at most in one of the sets $A_{i}$. If $h^{\prime} \notin \sum_{i=1}^{r} A_{i}$, then

$$
2 \varepsilon_{0}(K+1)^{2 R}<\sum_{k=1}^{r}\left\|\alpha\left(A_{k}\right)-\alpha(0)\right\| \leq \operatorname{Var}_{\alpha-\alpha(0)}\left(U_{N}-h^{\prime}\right)<\varepsilon_{0}
$$

which is a contradiction. On the other hand, if $h^{\prime} \in A_{m}$, then there exist disjoint sets $C_{1}, C_{2}, \ldots, C_{n}(n \leq R)$ of the class of sets $\mathcal{K}$ such that

$$
A_{m}=\left\{h^{\prime}\right\}+\sum_{k=1}^{n} C_{k}
$$


Now, let $\alpha$ be multiplicative. Applying Lemma 4 for $B_{2}=A_{m}, B_{1}=0$, we get

$$
\left\|e-\alpha\left(A_{m}\right)\right\| \leq(K+1)^{2 R}\left(\sum_{k=1}^{n} \operatorname{Var}_{\alpha-e}\left(C_{k}\right)+\left\|e-\alpha\left(h^{\prime}\right)\right\|\right) .
$$

Using this relation we conclude

$$
\begin{aligned}
2(K+1)^{2 R} \varepsilon_{0}< & \sum_{k=1}^{r}\left\|e-\alpha\left(A_{k}\right)\right\| \\
= & \sum_{k \neq m}\left\|e-\alpha\left(A_{k}\right)\right\|+\left\|e-\alpha\left(A_{m}\right)\right\| \\
\leq & (K+1)^{2 R}\left(\sum_{k \neq m}\left\|e-\alpha\left(A_{k}\right)\right\|+\sum_{k=1}^{n} \operatorname{Var}_{\alpha-e}\left(C_{k}\right)\right. \\
& \left.+\left\|e-\alpha\left(h^{\prime}\right)\right\|\right) \\
\leq & (K+1)^{2 R}\left(\operatorname{Var}_{\alpha-e}\left(U_{N}-h^{\prime}\right)+\left\|e-\alpha\left(h^{\prime}\right)\right\|\right) \\
\leq & 2 \varepsilon_{0}(K+1)^{2 R} .
\end{aligned}
$$

If $\alpha$ is additive, then

$$
\begin{aligned}
2(K+1)^{2 R} \varepsilon_{0} & <\sum_{k=1}^{r}\left\|\alpha\left(A_{k}\right)\right\| \\
& =\sum_{k \neq m}\left\|\alpha\left(A_{k}\right)\right\|+\left\|\alpha\left(A_{m}\right)\right\| \\
& \leq \sum_{k \neq m}\left\|\alpha\left(A_{k}\right)\right\|+\sum_{k=1}^{n}\left\|\alpha\left(C_{k}\right)\right\|+\left\|\alpha\left(h^{\prime}\right)\right\| \\
& \leq \operatorname{Var}_{\alpha}\left(U_{N}-h^{\prime}\right)+\left\|\alpha\left(h^{\prime}\right)\right\| \\
& \leq 2 \varepsilon_{0} \leq 2 \varepsilon_{0}(K+1)^{2 R} .
\end{aligned}
$$

In both cases we arrived at contradictions, hence our lemma is proved.

Lemma 6 If $\alpha(B)(\alpha(B) \in \mathcal{B}, B \in \mathcal{K})$ is a set function of bounded variation, then for every system $B_{1}, B_{2}, \ldots, B_{r}$ of disjoint sets of $\mathcal{K}$

$$
\left\|\prod_{k=1}^{r}\left(e+\alpha\left(B_{k}\right)\right)\right\| \leq K_{1}
$$

where $K_{1}$ is a constant.

ProOF. Let us select from the above product those $\alpha\left(B_{k}\right)$ 's for which $\left\|\alpha\left(B_{k}\right)\right\| \geq \frac{1}{2}$. The number of these elements is at most $N_{1}=\left[2 \operatorname{Var}_{\alpha}(H)\right]$. After this we form a maximal 
number of groups of the remaining $\alpha\left(B_{i}\right)$ 's such that in every group the sum of the norms fall in the interval $\left[\frac{1}{4}, \frac{1}{2}\right]$. The number of these groups is at most $N_{2}=\left[4 \operatorname{Var}_{\alpha}(H)\right]$. The sum of the norms of the remaining elements does not exceed $\frac{1}{4}$. Hence,

$$
\left\|\prod_{k=1}^{r}\left(e+\alpha\left(B_{k}\right)\right)\right\| \leq 2^{N_{2}+1}\left(\operatorname{Var}_{\alpha}(H)+1\right)^{N_{1}}=K_{1} .
$$

Lemma 7 Let us suppose that the set function $\alpha(A)(A \in \mathcal{K})$ with values in the Banach algebra $\mathcal{B}$ is multiplicative (and additive, resp.), $K=\operatorname{Var}_{\alpha-\alpha(0)}(H)<\infty$ and $\alpha(A)-\alpha(0)$ is $v$-continuous. If $B_{1}, B_{2} \ldots$ is a sequence of sets of $\mathcal{K}, h \in B_{k}(k=1,2, \ldots), \lim _{k \rightarrow \infty} d\left(B_{k}\right)=$ 0 , then

$$
\lim _{k \rightarrow \infty}\left\|\alpha\left(B_{k}\right)-\alpha(h)\right\|=0
$$

ProOF. Let $B_{k}=\{h\}+\sum_{i=1}^{R} C_{i}^{(k)}, C_{i}^{(k)} \in \mathcal{K}(i=1,2, \ldots, R ; k=1,2, \ldots)$ (we may always choose $R$ such sets since if we had $r<R$, then we should complete this system by $R-r$ void sets) and let $\alpha$ be multiplicative. Applying Lemma 6 for $\alpha-e$ instead of $\alpha$, moreover, using the inequality (1) we obtain

$$
\left\|\alpha\left(B_{k}\right)-\alpha(h)\right\| \leq K_{1}^{2} \sum_{i=1}^{R}\left\|\alpha\left(C_{i}^{(k)}\right)-e\right\| \leq K_{1}^{2} \sum_{i=1}^{R} \operatorname{Var}_{\alpha-e}\left(C_{i}^{(k)}\right) .
$$

Since $\lim _{k \rightarrow \infty} C_{i}^{(k)}=0(i=1,2, \ldots, R)$ and $\alpha(A)-e$ is $v$-continuous,

$$
\lim _{k \rightarrow \infty} \operatorname{Var}_{\alpha-e}\left(C_{i}^{(k)}\right)=0 \quad(i=1,2, \ldots, R)
$$

which proves the assertion.

Let us now consider the case of an additive $\alpha$. Since $\alpha(0)=0$, we get

$$
\left\|\alpha\left(B_{k}\right)-\alpha(h)\right\|=\left\|\sum_{i=1}^{R} \alpha\left(C_{i}^{(k)}\right)\right\| \leq \sum_{i=1}^{R} \operatorname{Var}_{\alpha}\left(C_{i}^{(k)}\right) .
$$

The right-hand side tends to 0 when $k \rightarrow \infty$, hence our statement is completely proved.

\section{$\S 3$. The additive total}

In this $\S$ our purpose is to prove the following

TheOREm 1 Let $f(A) \cdot(f(A) \in \mathcal{B})$ be a multiplicative set function defined on the elements of the class of sets $\mathcal{K}$. Suppose that $K=\operatorname{Var}_{f-e}(H)$ is finite and the set function $f(A)-e$ is $v$-continuous. In this case the additive total

$$
g(B)=\mathrm{S}_{B}(f(d A)-e)
$$


exists for every $B \in \mathcal{K}$ and

$$
\operatorname{Var}_{g}(B) \leq \operatorname{Var}_{f-e}(B)
$$

Proof. For the proof of the Theorem we need two lemmas.

Lemma 8 For every $h \in H$ and every $\varepsilon>0$ there can be found a $\delta>0$ such that if $A_{1}, A_{2}, \ldots, A_{r}$ are pairwise disjoint sets of the class of sets $\mathcal{K}$ with the property that $A=\sum_{k=1}^{r} A_{k} \in \mathcal{K}, h \in A$ and $d(A) \leq \delta$, then

$$
\left\|f(A)-e-\sum_{k=1}^{r}\left(f\left(A_{k}\right)-e\right)\right\| \leq \varepsilon
$$

Proof OF Lemma 8 Let $\delta$ be such a number that satisfies the conditions in Lemma 5 for $\frac{\varepsilon}{4 R(K+1)^{4 R}}$ instead of $\varepsilon$. The number $\delta$ can be chosen so small that the sphere with the centre $h$ and the radius $\delta$ does not contain an $h^{\prime} \in H\left(h^{\prime} \neq h\right)$ with $\left\|f\left(h^{\prime}\right)-e\right\|>$ $\frac{\varepsilon}{4 R(K+1)^{4 R}}$. We choose furthermore $\delta$ so small that $\|f(B)-f(h)\| \leq \frac{\varepsilon}{4}$ if $h \in B \in \mathcal{K}$, $d(B) \leq \delta$. By Lemma 7 this is always possible. Let $h \in A_{l}$. Obviously

$$
\begin{aligned}
\left\|f(A)-e-\sum_{k=1}^{r}\left(f\left(A_{k}\right)-e\right)\right\| & \leq\left\|f(A)-f\left(A_{l}\right)\right\|+\operatorname{Var}_{f-e}(A-h) \\
& \leq\|f(A)-f(h)\|+\left\|f\left(A_{l}\right)-f(h)\right\|+\operatorname{Var}_{f-e}(A-h) .
\end{aligned}
$$

Let $C_{1}, C_{2}, \ldots, C_{n}(n \leq R)$ be a system of disjoint sets of $\mathcal{K}$ for which $A-\{h\}=\sum_{k=1}^{n} C_{k}$. According to Lemmas 4 and 5

$$
\operatorname{Var}_{f-e}(A-h) \leq(K+1)^{2 R} \sum_{k=1}^{n} \operatorname{Var}_{f-e}\left(C_{k}\right) \leq(K+1)^{2 R} \frac{n \varepsilon}{2 R(K+1)^{2 R}} \leq \frac{\varepsilon}{2}
$$

On the other hand, we have chosen $\delta$ in such a way that

$$
\|f(A)-f(h)\| \leq \frac{\varepsilon}{4}, \quad\left\|f\left(A_{l}\right)-f(h)\right\| \leq \frac{\varepsilon}{4},
$$

hence our lemma is proved.

Lemma 9 Let $B \in \mathcal{K}$. To every $\varepsilon>0$ there can be found a number $\delta>0$ such that if $A_{1}, A_{2}, \ldots, A_{r}\left(A_{k} \in \mathcal{K} ; k=1,2, \ldots, r\right)$ is a system of disjoint subsets of $B$, $\max _{1 \leq k \leq r} d\left(A_{k}\right) \leq \delta$ and $A_{k}^{(1)}, A_{k}^{(2)}, \ldots, A_{k}^{\left(r_{k}\right)}$ is a decomposition of the set $A_{k}$ into pairwise disjoint sets of $\mathcal{K}$, then

$$
\sum_{k=1}^{r}\left\|f\left(A_{k}\right)-e-\sum_{i=1}^{r_{k}}\left(f\left(A_{k}^{(i)}\right)-e\right)\right\| \leq \varepsilon
$$


Proof of Lemma 9. We may suppose that the sets $A_{k}^{(i)}$ are so numbered that if $\mathfrak{z}_{k}=\left\{A_{k}^{(1)}, A_{k}^{(2)}, \ldots, A_{k}^{\left(r_{k}\right)}\right\}$, then $\mathcal{P}\left(\mathfrak{z}_{k}\right)=\left(A_{k}^{(1)}, A_{k}^{(2)}, \ldots, A_{k}^{\left(r_{k}\right)}\right)$. Since the variation of $f(A)-e$ is equal to $K$, the number of the points $h \in H$ for which $\|f(h)-e\|>\frac{\varepsilon}{8 K(K+1)^{2 R}}$ is at most $\frac{8 K^{2}}{\varepsilon}(K+1)^{2 R}$. If such a point exists in $B$, then we renumber the sets $A_{k}$ so that those sets, which contain these points, be $A_{1}, A_{2}, \ldots, A_{l}\left(l \leq r, l \leq \frac{8 K^{2}(K+1)^{2 R}}{\varepsilon}\right)$. By Lemma $8 \delta$ can be chosen so small that

$$
\left\|f\left(A_{k}\right)-e-\sum_{i=1}^{r_{k}}\left(f\left(A_{k}^{(i)}\right)-e\right)\right\| \leq \frac{\varepsilon}{2} \frac{\varepsilon}{8 K^{2}(K+1)^{2 R}} \leq \frac{\varepsilon}{2 l} \quad(k=1,2, \ldots, l) .
$$

If there are sets which do not contain such points and these are $A_{l+1}, \ldots, A_{r}$, then we choose $\delta$ so small that besides (10) the following inequality holds:

$$
\operatorname{Var}_{f-e}\left(A_{k}\right) \leq \frac{\varepsilon}{4 K} \quad(k=l+1, l+2, \ldots, r) .
$$

By Lemma 5 this is possible since the sets $A_{l+1}, A_{l+2}, \ldots, A_{r}$ do not contain points $h$ with $\|f(h)-e\|>\frac{\varepsilon}{8 K(K+1)^{2 R}}$. Applying the inequality (4) for $f_{i}=f\left(A_{k}^{(i)}\right)-e(i=$ $1,2, \ldots, r_{k}$ ) we get (we may suppose that $0<\varepsilon \leq 2 K$ )

$$
\begin{aligned}
\left\|f\left(A_{k}\right)-e-\sum_{i=1}^{r_{k}}\left(f\left(A_{k}^{(i)}\right)-e\right)\right\| & =\left\|\prod_{i=1}^{r_{k}} f\left(A_{k}^{(i)}\right)-e-\sum_{i=1}^{r_{k}}\left(f\left(A_{k}^{(i)}\right)-e\right)\right\| \\
& \leq 2\left(\sum_{i=1}^{r_{k}}\left\|f\left(A_{k}^{(i)}\right)-e\right\|\right)^{2} \\
& \leq 2\left(\operatorname{Var}_{f-e}\left(A_{k}\right)\right)^{2} \leq 2 \frac{\varepsilon}{4 K} \operatorname{Var}_{f-e}\left(A_{k}\right) .
\end{aligned}
$$

Our statement follows from the inequalities (10) and (11).

After these preparations we can complete the proof of Theorem 1 . We point out that if $B \in \mathcal{K}$ is a fixed set and $\mathfrak{z}_{n}=\left\{A_{n k}\right\}$ is a sequence of decompositions of $B$ with $\lim _{n \rightarrow \infty} \max _{k} d\left(A_{n k}\right)=0$, then the sequence

$$
\sum_{k}\left(f\left(A_{n k}\right)-e\right)
$$

satisfies the Cauchy's convergence criterion. For this purpose we consider two decompositions $A_{1}, A_{2}, \ldots, A_{r}$ and $A_{1}^{\prime}, A_{2}^{\prime}, \ldots, A_{r^{\prime}}^{\prime}$, of the set $B$ into pairwise disjoint sets of $\mathcal{K}$ with $\max _{1 \leq k \leq r} d\left(A_{k}\right) \leq \delta, \max _{1 \leq k \leq r^{\prime}} d\left(A_{k}^{\prime}\right) \leq \delta$ where $\delta$ is the same number as in Lemma 9 . If $\delta$ is so small that (9) is satisfied with $\frac{\varepsilon}{2}$ instead of $\varepsilon$, then, considering the superposition of the two decompositions, a well-known argument shows that

$$
\left\|\sum_{k=1}^{r}\left(f\left(A_{k}\right)-e\right)-\sum_{k=1}^{r^{\prime}}\left(f\left(A_{k}^{\prime}\right)-e\right)\right\| \leq \varepsilon
$$

and thus $\mathrm{S}_{B}(f(d A)-e)$ exists. The relation (7) can be proved in an obvious way. 


\section{$\S 4$. The multiplicative total}

In this section we prove the following

TheOREm 2 Let $g(B)(g(B) \in \mathcal{B})$ be an additive set function defined on the elements of the class of sets $\mathcal{K}$. Suppose that $\operatorname{Var}_{g}(H)<\infty$ and the set function $g(B)(B \in \mathcal{K})$ is $v$-continuous. In this case for any permutation function $\mathcal{P}$ and for every $A \in \mathcal{K}$ the total

$$
f(A)={ }_{\mathcal{P}} \prod_{A}(g(d B)+e)
$$

exists and

$$
\operatorname{Var}_{f-e}(A) \leq L \operatorname{Var}_{g}(A),
$$

where $L$ is a constant independent of the set $A$.

Proof. First we prove two lemmas.

LEMma 10 For every $h \in H$ and $\varepsilon>0$ there can be found $a \delta>0$ such that if $B_{1}, B_{2}, \ldots, B_{r}$ are disjoint sets of $\mathcal{K}$ with $B=\sum_{k=1}^{r} B_{k} \in \mathcal{K}, h \in B, d(B) \leq \delta$, then

$$
\left\|g(B)+e-\prod_{k=1}^{r}\left(g\left(B_{k}\right)+e\right)\right\| \leq \varepsilon .
$$

Proof of Lemma 10. Let us suppose that $h \in B_{l}$. Using Lemma 6 and the inequality (1) it follows that

$$
\begin{aligned}
& \left\|g(B)+e-\prod_{k=1}^{r}\left(g\left(B_{k}\right)+e\right)\right\| \\
& =\left\|e(g(B)+e) e-\prod_{k=1}^{l-1}\left(g\left(B_{k}\right)+e\right)\left(g\left(B_{l}\right)+e\right) \prod_{k=l+1}^{r}\left(g\left(B_{k}\right)+e\right)\right\| \\
& \quad \leq K_{1}^{2}\left(\left\|\prod_{k=1}^{l-1}\left(g\left(B_{k}\right)+e\right)-e\right\|+\left\|\prod_{k=l+1}^{r}\left(g\left(B_{k}\right)+e\right)-e\right\|+\left\|g(B)-g\left(B_{l}\right)\right\|\right) .
\end{aligned}
$$

Let $\varepsilon \leq K_{1}^{2}$. By Lemmas 5 and 7 (taking into account the remark made after Lemma 4) $\delta$ can be chosen so that

$$
\operatorname{Var}_{g}(C-h) \leq \frac{\varepsilon}{4 K_{1}^{2}}
$$

moreover

$$
\|g(C)-g(h)\| \leq \frac{\varepsilon}{4 K_{1}^{2}}
$$


where $C \in \mathcal{K}, h \in C, d(C)<\delta$; then from (3) and (14) it follows

$$
\begin{aligned}
& \left\|\prod_{k=1}^{l-1}\left(g\left(B_{k}\right)+e\right)-e\right\|+\left\|\prod_{k=l+1}^{r}\left(g\left(B_{k}\right)+e\right)-e\right\| \\
& \quad \leq 2 \sum_{k=1}^{l-1}\left\|g\left(B_{k}\right)\right\|+2 \sum_{k=l+1}^{r}\left\|g\left(B_{k}\right)\right\| \leq 2 \operatorname{Var}_{g}(B-h) \leq \frac{\varepsilon}{2 K_{1}^{2}}
\end{aligned}
$$

relation (15) implies that

$$
\left\|g(B)-g\left(B_{l}\right)\right\| \leq \frac{\varepsilon}{2 K_{1}^{2}}
$$

hence our assertion holds.

Lemma 11 Let $A \in \mathcal{K}$. To every $\varepsilon>0$ there can be found a number $\delta>0$ such that if $B_{1}, B_{2}, \ldots, B_{r}\left(B_{k} \in \mathcal{K} ; k=1,2, \ldots, r\right)$ is a system of disjoint subsets of $A$ and $B_{k}^{(1)}, B_{k}^{(2)}, \ldots, B_{k}^{\left(r_{k}\right)}$ is a decomposition of the set $B_{k}$ into pairwise disjoint sets of $\mathcal{K}$, $d\left(B_{k}\right) \leq \delta$, then

$$
\sum_{k=1}^{r}\left\|e+g\left(B_{k}\right)-\prod_{i=1}^{r_{k}}\left(e+g\left(B_{k}^{(i)}\right)\right)\right\| \leq \varepsilon
$$

Proof of Lemma 11. If for a $k$ we have $\operatorname{Var}_{g}\left(B_{k}\right) \leq \frac{1}{2}$, then by the inequality (4) it follows that

$$
\left\|e+g\left(B_{k}\right)-\prod_{i=1}^{r_{k}}\left(e+g\left(B_{k}^{(i)}\right)\right)\right\| \leq 2\left(\sum_{i=1}^{r_{k}}\left\|g\left(B_{k}^{(i)}\right)\right\|\right)^{2} \leq 2\left(\operatorname{Var}_{g}\left(B_{k}\right)\right)^{2} .
$$

The remaining part of the proof can be accomplished by the aid of Lemma 10 in a similar way as we have proved Lemma 9 by the aid of Lemma 8 .

In order to complete the proof of Theorem 2 let us consider the decompositions $\left\{B_{1}, B_{2}, \ldots, B_{r}\right\},\left\{B_{1}^{\prime}, B_{2}^{\prime}, \ldots, B_{r^{\prime}}^{\prime}\right\}$ of the set $A$ into pairwise disjoint sets of $\mathcal{K}$ satisfying $\max _{1 \leq k \leq r} d\left(B_{k}\right) \leq \delta, \max _{1 \leq k \leq r^{\prime}} d\left(B_{k}^{\prime}\right) \leq \delta$ where $\delta$ is a number fixed in Lemma 11. Let $\left\{B_{1}^{\prime \prime}, B_{2}^{\prime \prime}, \ldots, B_{r^{\prime \prime}}^{\prime \prime}\right\}$ denote the superposition of these two decompositions. If $\delta$ is so small that the inequality (16) holds for $\frac{\varepsilon}{2 K_{1}^{2}}$ instead of $\varepsilon$, then

$$
\begin{aligned}
\left\|\prod_{k=1}^{r}\left(g\left(B_{i_{k}}\right)+e\right)-\prod_{k=1}^{r^{\prime}}\left(g\left(B_{j_{k}}^{\prime}\right)+e\right)\right\| \leq & \left\|\prod_{k=1}^{r}\left(g\left(B_{i_{k}}\right)+e\right)-\prod_{k=1}^{r^{\prime \prime}}\left(g\left(B_{n_{k}}^{\prime \prime}\right)+e\right)\right\| \\
& +\left\|\prod_{k=1}^{r^{\prime}}\left(g\left(B_{j_{k}}^{\prime}\right)+e\right)-\prod_{k=1}^{r^{\prime \prime}}\left(g\left(B_{n_{k}}^{\prime \prime}\right)+e\right)\right\|
\end{aligned}
$$




$$
\begin{aligned}
\leq & K_{1}^{2} \sum_{k=1}^{r}\left\|g\left(B_{i_{k}}\right)+e-\prod_{B_{n_{s}}^{\prime \prime} \subseteq B_{i_{k}}}\left(g\left(B_{n_{s}}^{\prime \prime}\right)+e\right)\right\| \\
& +K_{1}^{2} \sum_{k=1}^{r^{\prime}}\left\|g\left(B_{j_{k}}^{\prime}\right)+e-\prod_{B_{n_{s}}^{\prime \prime} \subseteq B_{j_{k}}^{\prime}}\left(g\left(B_{n_{s}}^{\prime \prime}\right)+e\right)\right\| \\
\leq & \frac{\varepsilon}{2}+\frac{\varepsilon}{2}=\varepsilon,
\end{aligned}
$$

where the sequences $i_{k}, j_{k}, n_{k}$ are fixed by the permutation function $\mathcal{P}$ and in the products

$\prod_{B_{n_{s}}^{\prime \prime} \subseteq B_{i_{k}}}, \prod_{B_{n_{s}}^{\prime \prime} \subseteq B_{j_{k}}^{\prime}}$ the factors are arranged according to the same order as they were in the first row. Hence the existence of

$$
f(A)={ }_{\mathcal{P}} \prod_{A}(g(d B)+e)
$$

follows in an obvious way.

The proof of (12) can be accomplished as follows. By making use of Lemma 1 we get

$$
\left\|\prod_{k=1}^{r_{s}}\left(g\left(B_{s}^{\left(i_{k}\right)}\right)+e\right)-e\right\| \leq K_{1}^{2} \sum_{k=1}^{r_{s}}\left\|g\left(B_{s}^{\left(i_{k}\right)}\right)\right\| \leq K_{1}^{2} \operatorname{Var}_{g}\left(B_{s}\right) .
$$

Hence it follows

$$
\left\|f\left(B_{s}\right)-e\right\| \leq K_{1}^{2} \operatorname{Var}_{g}\left(B_{s}\right)
$$

and

$$
\sum_{s=1}^{r}\left\|f\left(B_{s}\right)-e\right\| \leq K_{1}^{2} \sum_{s=1}^{r} \operatorname{Var}_{g}\left(B_{s}\right) \leq K_{1}^{2} \operatorname{Var}_{g}(A)
$$

whence

$$
\operatorname{Var}_{f-e}(A) \leq K_{1}^{2} \operatorname{Var}_{g}(A)
$$

what was to be proved.

\section{$\S 5$. Connection between the additive and multiplicative to- tals}

According to Theorems 1 and 2 the indefinite additive (and multiplicative) total of a multiplicative (and additive, resp.) and $v$-continuous set function is also $v$-continuous. Hence it may be a starting function of further totalization. In this $\S$ we prove that the latter total (with respect to some permutation function) coincides with the original one. First we prove 
Theorem 3 Let $g(B)(g(B) \in \mathcal{B})$ be an additive set function defined on the elements of the class of sets $\mathcal{K}$ and satisfying the conditions of Theorem 2. If $A \in \mathcal{K}$ and

$$
f(A)={ }_{\mathcal{P}} \prod_{A}(g(d B)+e)
$$

then the additive total of $f(A)-e$ exists in every $B \in \mathcal{K}$ and

$$
g(B)=\mathrm{S}_{B}(f(d A)-e)
$$

Proof. Let $B_{1}, B_{2}, \ldots, B_{r}$ be a system of disjoint sets of $\mathcal{K}$ with the property that $A=\sum_{k=1}^{r} B_{k} \in \mathcal{K}, \max _{1 \leq k \leq r} d\left(B_{k}\right) \leq \delta$. Relation (16) implies that in case of a small $\delta$

$$
\sum_{k=1}^{r}\left\|e+g\left(B_{k}\right)-f\left(B_{k}\right)\right\| \leq \frac{\varepsilon}{2} .
$$

If we introduce the notation

$$
g^{\prime}(B)=\mathrm{S}_{B}(f(d A)-e),
$$

then by (9), choosing $\delta$ small enough, we obtain

$$
\left.\sum_{k=1}^{r} \| g^{\prime}\left(B_{k}\right)-f\left(B_{k}\right)-e\right) \| \leq \frac{\varepsilon}{2}
$$

Relations (19) and (20) imply

$$
\sum_{k=1}^{r}\left\|g\left(B_{k}\right)-g^{\prime}\left(B_{k}\right)\right\| \leq \varepsilon
$$

It follows that

$$
\left\|g(A)-g^{\prime}(A)\right\|=\left\|\sum_{k=1}^{r} g\left(B_{k}\right)-\sum_{k=1}^{r} g^{\prime}\left(B_{k}\right)\right\| \leq \sum_{k=1}^{r}\left\|g\left(B_{k}\right)-g^{\prime}\left(B_{k}\right)\right\| \leq \varepsilon
$$

and thus $g(A)=g^{\prime}(A)$.

A similar theorem can be proved if we start from a multiplicative set function. In advance we remind of Definition 3 that to every multiplicative set function a permutation function is attached. Our statement is expressed in

TheOREM 4 Let $f(A)(f(A) \in \mathcal{B})$ be a multiplicative set function defined on the elements of the class of sets $\mathcal{K}$ and satisfying the conditions of Theorem 1 . If $B \in \mathcal{K}$ and

$$
g(B)=\mathrm{S}_{B}(f(d A)-e),
$$

moreover $\mathcal{P}$ is a permutation function attached to $f$, then ${ }_{\mathcal{P}} \prod_{A}(g(d B)+e)$ exists for every $A \in \mathcal{K}$ and

$$
f(A)={ }_{\mathcal{P}} \prod_{A}(g(d B)+e)
$$


Proof. Let us introduce the notation

$$
f^{\prime}(A)={ }_{\mathcal{P}} \prod_{A}(g(d B)+e) .
$$

If $A_{1}, A_{2}, \ldots, A_{r}$ is a system of disjoint sets of $\mathcal{K}$ with the property that $\max _{1 \leq k \leq r} d\left(A_{k}\right) \leq \delta$ and $B=\sum_{k=1}^{r} A_{k}$, then by formula (9) (for a small $\delta$ )

$$
\sum_{k=1}^{r}\left\|f\left(A_{k}\right)-e-g\left(A_{k}\right)\right\| \leq \frac{\varepsilon}{2} .
$$

According to (16) we get furthermore that if $\delta$ is small enough, then

$$
\sum_{k=1}^{r}\left\|e+g\left(A_{k}\right)-f^{\prime}\left(A_{k}\right)\right\| \leq \frac{\varepsilon}{2}
$$

Hence, by (23) and (24),

$$
\sum_{k=1}^{r}\left\|f\left(A_{k}\right)-f^{\prime}\left(A_{k}\right)\right\| \leq \varepsilon
$$

Let $\mathcal{P}(\mathfrak{z})=\left(A_{i_{1}}, A_{i_{2}}, \ldots, A_{i_{r}}\right)$ be the permutation corresponding to $\mathfrak{z}=\left\{A_{1}, A_{2}, \ldots, A_{r}\right\}$. By Lemma 6 there exists a $K_{1}$ such that

$$
\begin{aligned}
\left\|\prod_{k=1}^{j} f\left(A_{i_{k}}\right)\right\| & \left.=\| \prod_{k=1}^{j}\left[f\left(A_{i_{k}}\right)-e\right)+e\right] \| \leq K_{1}, \\
\left\|\prod_{k=j}^{r} f^{\prime}\left(A_{i_{k}}\right)\right\| & \left.=\| \prod_{k=j}^{r}\left[f^{\prime}\left(A_{i_{k}}\right)-e\right)+e\right] \| \leq K_{1} .
\end{aligned}
$$

Taking into account (25) and (26) and applying Lemma 1 we obtain

$$
\left\|f(A)-f^{\prime}(A)\right\|=\left\|\prod_{k=1}^{r} f\left(A_{i_{k}}\right)-\prod_{k=1}^{r} f^{\prime}\left(A_{i_{k}}\right)\right\| \leq K_{1}^{2} \varepsilon,
$$

hence $f(A)=f^{\prime}(A)$.

The last two theorems show that the indefinite total of an additive (and multiplicative, resp.) set function entirely determines the original set function.

\section{$\S$ 6. Examples}

\section{The weighted random point distribution}

Let us consider a random selection of a finite number of points of $H$ where each selected point is weighted with a (positive or negative) integer. We suppose that the sum of 
the weights in a set $A \in \mathcal{K}$ is a random variable which we denote by $\xi(A)$. We suppose furthermore that if $A_{1}, A_{2}, \ldots, A_{r}$ are disjoint sets of $\mathcal{K}$, then the random variables $\xi\left(A_{1}\right), \xi\left(A_{2}\right), \ldots, \xi\left(A_{r}\right)$ are independent. Let us introduce the notation

$$
P_{k}(A)=\mathbb{P}(\xi(A)=k) .
$$

The sequences $P(A)=\left(\ldots, P_{-2}(A), P_{-1}(A), P_{0}(A), P_{1}(A), P_{2}(A), \ldots\right)$ are elements of the Banach algebra $\mathcal{B}$ of the sequences the corresponding series of which are absolutely convergent with the norm of the sum of the absolute values. If the product of two elements $\left(\ldots, a_{-1}, a_{0}, a_{1}, \ldots\right),\left(\ldots, b_{-1}, b_{0}, b_{1}, \ldots\right)$ of $\mathcal{B}$ is the convolution

$$
\left(\sum_{k=-\infty}^{\infty} a_{n-k} b_{k} ; \quad n=0, \pm 1, \pm 2, \ldots\right),
$$

then $\mathcal{B}$ is commutative and has as unity element that one for which the member corresponding to the index 0 is equal to 1 and the others are 0 . In this case $P(A)$ is a multiplicative set function. We may establish a more stronger statement, namely

$$
P(A)=P\left(A_{1}\right) P\left(A_{2}\right) \ldots=\lim _{n \rightarrow \infty} \prod_{k=1}^{n} P\left(A_{k}\right)
$$

if $A_{i} \in \mathcal{K}(i=1,2, \ldots), A_{i} A_{k}=0$ for $i \neq k$ and $A=\sum_{i=1}^{\infty} A_{i} \in \mathcal{K}$.

We shall show that $P(A)-e$ is of bounded variation and $v$-continuous. Since

$$
\|P(A)-e\|=\sum_{k \neq 0} P_{k}(A)+1-P_{0}(A)=2\left(1-P_{0}(A)\right),
$$

we have to prove the assertion for the real-valued set function $1-P_{0}(A)$. Let us first extend the definition of $\xi(A)$ to $\mathcal{R}(\mathcal{K})$ which is the smallest ring ${ }^{4}$ containing $\mathcal{K}$. Let $A_{1}, A_{2}, \ldots$ be a sequence of disjoint sets of $\mathcal{R}(\mathcal{K})$. Since we have selected only a finite number of points from $H$, the sequence of independent random variables

$$
\sum_{k=1}^{\infty} \xi\left(A_{k}\right)
$$

converges with probability 1. Hence, by the three series theorem of Kolmogorov or simply by the Borel-Cantelli lemma,

$$
\sum_{k=1}^{\infty}\left(1-P_{0}\left(A_{k}\right)\right)<\infty .
$$

On the other hand, $1-P_{0}(A)$ is completely subadditive in the following sense: if $A_{1}, A_{2}, \ldots$ is a sequence of disjoint sets of $\mathcal{K}$ for which $A=\sum_{k=1}^{\infty} A_{k} \in \mathcal{R}(\mathcal{K})$, then

$$
1-P_{0}(A) \leq \sum_{k=1}^{\infty}\left(1-P_{0}\left(A_{k}\right)\right) .
$$

\footnotetext{
${ }^{4} \mathrm{~A}$ class of sets $\mathcal{R}$ is called a ring if $A+B \in \mathcal{R}, A-B \in \mathcal{R}$, provided that $A \in \mathcal{R}, B \in \mathcal{R}$. This extension is obviously possible and relation (29) will be satisfied also in $\mathcal{R}(\mathcal{K})$.
} 
In fact, the event $\xi(A) \neq 0$ implies that at least one of $\xi\left(A_{1}\right) \neq 0, \xi\left(A_{2}\right) \neq 0, \ldots$ occurs. Hence, by Lemma 4 of [5], $1-P_{0}(A)$ is of bounded variation and by Lemma 1 of [5] $\operatorname{Var}_{1-P_{0}}(A)(A \in \mathcal{R}(\mathcal{K}))$ is a bounded measure. This last property implies that $1-P_{0}(A)$ is $v$-continuous. Thus the additive total

$$
Q(B)=\mathrm{S}_{B}(P(d A)-e)
$$

exists for every $B \in \mathcal{K}$. This implies obviously the existence of the totals

$$
\begin{aligned}
& Q_{0}(B)=\mathbf{S}_{B}\left(P_{0}(d A)-1\right), \\
& Q_{k}(B)=\mathbf{S}_{B} P_{k}(d A) \quad(k \neq 0) .
\end{aligned}
$$

Since the convergence in (30) holds in the norm, it follows that

$$
Q_{0}(B)=-\sum_{k \neq 0} Q_{k}(B)
$$

Moreover, the relation

$$
Q_{k}(B) \leq-Q_{0}(B) \leq \operatorname{Var}_{1-P_{0}}(B) \quad(B \in \mathcal{K})
$$

implies that with $\operatorname{Var}_{1-P_{0}}(A)$ the additive set functions $-Q_{0}(B), Q_{k}(B)(k= \pm 1, \pm 2, \ldots)$ are also bounded measures. Finally, if $h \in H$, then

$$
\mathrm{S}_{\{h\}}\left(1-P_{0}(d A)\right)=1-P_{0}(\{h\}) \leq 1,
$$

hence

$$
-Q_{0}(\{h\}) \leq 1
$$

It is interesting to write all the solutions of (29) in a closed form, provided that the sequence $P(A)$ is a probability distribution. This can be done as follows. We start from a sequence of bounded measures $-Q_{0}(B), Q_{k}(B)(k= \pm 1, \pm 2, \ldots)$ satisfying (32) and (33). Then every solution of $(29)$ can be represented as

$$
P(A)=\prod_{A}(e+Q(d B)) \quad(A \in \mathcal{K}),
$$

where $Q(B)=\left(\ldots, Q_{-1}(B), Q_{0}(B), Q_{1}(B), \ldots\right)$. In fact, if $P(A)$ is a solution of $(29)$, then the $Q(B)$ defined by (30) has the mentioned properties, hence by Theorem 4 (34) holds. Conversely, if $Q(B)$ has the mentioned properties, then by Theorem 2 (34) exists and the properties of $Q(B)$ imply that $P(A)$ is a probability distribution for every $A \in \mathcal{K}$ and (29) holds. By Theorems 3 and 4 the correspondence between the set functions $P(A)$ and $Q(B)$ is one-to-one.

It is not difficult to see that $Q_{k}(B)$ is the expected number of points weighted with $k$ in the set $B$. (This can be deduced immediately from the results of [6] too.) If there are no $h \in H$ such that $1-P_{0}(\{h\})>0$, then $P(A)$ is a compound Poisson distribution, i.e. it has the characteristic function

$$
\exp \sum_{k \neq 0} Q_{k}(A)\left(e^{i k u}-1\right)
$$


If all the random points are weighted by 1 , then from (35) we obtain a Poisson distribution

$$
\exp Q_{1}(A)\left(e^{i u}-1\right) \text {. }
$$

The proof of (35) can be accomplished with the aid of the relation (34). Similar statements are proved in $[6]$.

\section{The linear integer-valued Markov process}

Let $\xi_{t}$ be a Markov process on the linear interval $a \leq t \leq b$. We suppose that $\xi_{t}$ can take on only the values $1,2, \ldots, N$. Let $\mathcal{K}$ be the semi-ring of all the subintervals of $[a, b]$ (we permit here closed, open, semi-closed, degenerated intervals equally). If the right-hand and left-hand limits of the transition probability matrices $P\left(t_{1}, t_{2}\right)$ exists when $t_{1}$ or $t_{2}$ tend to a limit, then we can correspond to every $I \in \mathcal{K}$ a matrix $P(I) . P(I)$ is an element of the (non-commutative) Banach algebra of $N$-rowed quadratic matrices, where the norm is the maximal value among the absolute column-sums. Obviously $\mathcal{B}$ has a unite element. The set function $P(I)$ is then multiplicative relative to the natural permutation of linear intervals. Hence, if $P(I)$ is of bounded variation and $v$-continuous, then we can write the solution of the equation

$$
P\left(I_{1}+I_{2}\right)=P\left(I_{1}\right) P\left(I_{2}\right) \quad\left(I_{1} \in \mathcal{K}, I_{2} \in \mathcal{K}, I_{1}+I_{2} \in \mathcal{K}\right)
$$

in a closed form. We will not enter into the details since in the paper of DobRUŠIN [3] this is profoundly investigated under somewhat general assumptions. An analogous treatment can be given for the Markov processes having a countable number of possible states. To this question the author will return later.

Finally, I express my thank to Á. CsÁszÁr for his valuable remarks.

\section{References}

[1] Arley, N. (1948). On the Theory of Stochastic Processes and Their Applications to the Theory of Cosmic Radiation, New York.

[2] Birkhoff, G. (1937). On Product Integration, Journal of Math. and Phys., 16, 104-132.

[3] Dobrušin, R. L. (1953). Obobscsenie uravnenij Kolmogorova dlja markovszkih processzov sz konecsnüm csiszlom vozmozsnüh szosztojanij, Mat. Szbornik, 33, 566-596.

[4] Fréchet, M. (1938). Recherches théoriques modernes sur le calcul des probabilités. II, Paris, p. 210.

[5] PrÉKopA, A. (1956). Extension of Multiplicative Set Functions with Values in a Banach Algebra, Acta Math. Acad. Sci. Hung., 7, 201-213.

[6] Prékopa, A. (1957). On Poisson and Composed Poisson Stochastic Set Functions, Studia Math., 16 142-157. 
[7] Rasch, G. (1943). Zur Theorie und Anwendung des Produkintegrals, Journal f. reine u. angew. Math., 171, 65-119.

[8] Schlesinger, L. (1908). Vorlesungen über lineare Differentialgleichungen, Berlin.

[9] Schlesinger, L. (1922). Einführung in die Theorie der gewöhnlichen Differentialgleuchungen auf funktionentheoretischer Grundlage, Berlin.

[10] Schlesinger, L. (1931). Neue Grundlagen für einen Infinitesimalkalkül der Matrizen, Math. Zeitschrift, 33, 33-61.

[11] Schlesinger, L. (1932). Weitere Beiträge zum Infinitesimalkalkül der Matrizen, Math. Zeitschrift, 35, 485-501.

[12] Volterra, V. (1887). Sui fondamenti della teoria delle equazioni differenziali lineari, Mem. Soc. Ital. delle Scienze (3), 6, p. 107.

[13] Volterra, V. (1888). Sulla teoria delle equazioni differenziali lineari, Rendiconti del Circolo Mat. di Palermo, 3, 69-75.

[14] Volterra, V. (1902). Sui fondamenti della teoria delle equazioni differenziali lineari, Mem. Soc. Ital. delle Scienze (3), 12, 3-68. 